\title{
Utilidad del índice triglicéridos/HDL-C desde los primeros años de vida en el diagnóstico de síndrome metabólico en niños obesos
}

\author{
Utility of the triglycerides/HDL-C index from the first years of age in the diagnosis of metabolic syndrome \\ in obese children
}

Emilio Cabello 1,a; 2,b,c, Melissa Martínez ${ }^{1, a ; ~ 2, e}$ Ysel Cabrera $^{\text {1, 2,f, }}$, Susan Villafuerte ${ }^{1, a ; ~ 2, e}$, Isolda González 1,a; 2,d

\section{RESUMEN}

Objetivo: Determinar la utilidad del índice triglicéridos/HDL-C (TG/HDL-C) para el diagnóstico de síndrome metabólico (SM) en niños obesos de 2 a 14 años. Material y métodos: Estudio transversal tipo prueba de diagnóstico. Fueron incluidos 360 niños obesos exógenos (199M/161F), divididos en tres grupos etarios: 2 a $<6$ años, 6 a $<10$ años y 10 a 14 años. Se definió SM según los criterios de la International Diabetes Federation y se evaluó al índice TG/HDL-C como diagnóstico en dos puntos de corte: $\geq 2,32$ y $\geq 3,5$, en cada grupo etario. Se aplicó Chi cuadrado, considerándose significativo $\mathrm{p}<0,05$. Se determinó la sensibilidad, especificidad y valores predictivos positivo y negativo, para cada punto de corte. Resultados: La frecuencia de SM fue 15,79\% de 2 a $<6$ años, 20,25\% de 6 a $<10$ años, 19,63\% de 10 a 14 años. En los niños con SM el 97,1\% presentó HDL bajo, 83,8\% triglicéridos elevados. Se encontró diferencia significativa en la frecuencia del índice TG/HDL-C en ambos puntos de corte, entre los niños con y sin SM en todos los grupos etarios. La sensibilidad para ambos puntos de corte fue alta (86$100 \%$ ) y la especificidad fue mejor para el punto de corte $\geq 3,5$ (72-80\%). Conclusiones: El índice TG/HDL-C $\geq 3,5$ representaría un marcador sensible y específico para el diagnóstico de síndrome metabólico desde los primeros años de vida.

PALABRAS CLAVE: Índice TG/HDL-C, síndrome metabólico, niños. (Fuente: DeCS BIREME).

\section{SUMMARY}

Objective: To determine the utility of the triglycerides/HDL-C index for the diagnosis of metabolic syndrome (MS) in obese 2-14 years of age children. Methods: Cross-sectional diagnostic study. We included 360 exogenous obese children $(199 \mathrm{M} / 161 \mathrm{~F})$ divided in three age groups: 2 to $<6 ; 6$ to $<10$ and $10-14$ years. MS was defined according to the International Diabetes Federation and the TG/HDL-C index was evaluated using two cutoffs by age group, $\geq 2.32$ and $\geq 3.5$. Chi squared was used accepting a $p$ value $<0.05$ as statistically significant. Fir each cut-off we determined sensitivity, specificity and predictive values. Results: Frequency of MS was $15.79 \%$ in the 2 to $<6$ years age group; $20.25 \%$ in the 6 to $<10$ years age group and $19.63 \%$ in the $10-14$ years age group. Among children with

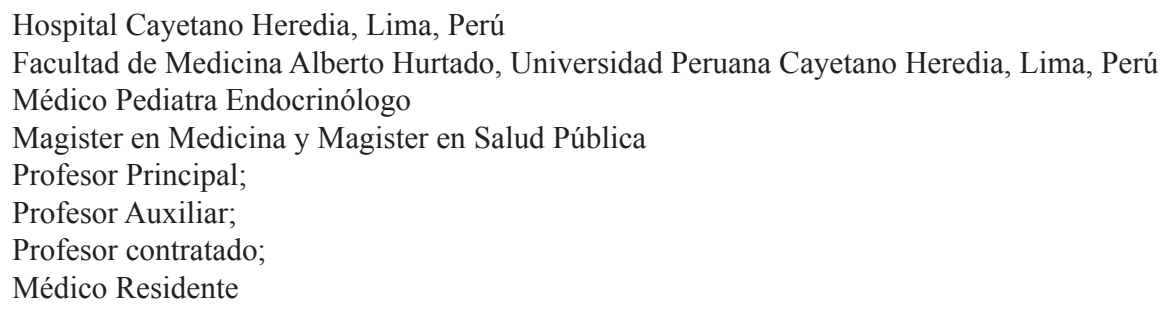


SM, 97.1\% had low HDL and $83.8 \%$ had elevated triglycerides. A statistically significant difference was found in the frequency of the TG/HDL-C index in children with or without MS in all age groups. Sensitivity for all cut-offs was high (86-100\%) and specificity was best for the cut-off of $\geq 3.5$ (72-80\%). Conclusions: The TG/HDL-C index is a sensitive and specific marker of MS from the first years of age.

KEYWORDS: TG / HDL-C ratio, metabolic syndrome, children. (Source: MeSH NLM).

\section{INTRODUCCIÓN}

La obesidad en los niños es un problema de salud pública en el Perú, que en las últimas décadas ha mostrado una tendencia creciente desde los primeros años de vida (1). Se asocia a diabetes mellitus tipo 2 (DM2) y enfermedad cardiovascular (ECV) en el adulto $(2,3)$. La excesiva acumulación de tejido adiposo, especialmente a nivel visceral sería el principal desencadenante de las alteraciones metabólicas del síndrome metabólico (SM) en niños y adolescentes (4). En su fisiopatología se incluyen mecanismos de resistencia a la insulina (RI), activación neurohormonal y activación de un proceso inflamatorio crónico $(5,6,7)$.

El exceso de tejido adiposo satura su capacidad de almacenamiento y disminuye la capacidad de la insulina para suprimir la lipólisis, ocasionando incremento de ácidos grasos libres circulantes, disminución de la captación de glucosa a nivel muscular, promoción de gluconeogénesis y lipogénesis, los que inducen el incremento de insulina para mantener un estado de normo glicemia (8). Asimismo, se estimula la secreción de TNF- $\alpha$ que produce fosforilación e inactivación de los receptores de insulina, incrementando los ácidos grasos libres e inhibiendo la liberación de adiponectina (9). Por otro lado, se estimula la producción de leptina, angiotensina II y de sustancias reactivas de oxígeno que conducen a daño endotelial y proliferación de fibroblastos que se manifestarán como hipertensión arterial, dislipidemia, DM2 y ECV $(10,11)$.

El diagnóstico de síndrome metabólico en niños $\mathrm{y}$ adolescentes es controversial, en tanto no existe consenso respecto a los criterios utilizados para su definición y a su utilidad clínica como predictor de DM2 y ECV en el adulto (12). Existen varias propuestas para su definición (13-17); lo que determina la variabilidad en las prevalencias encontradas. En nuestro medio, Cabello E y col. (18), comparando cuatro diferentes definiciones en niños de 4 a 14 años, encontraron menor prevalencia de SM utilizando los criterios de la International Diabetes Federation (IDF) respecto a Cook, Cruz y la Academia Americana de Pediatría (AAP).
Respecto a la relevancia de su identificación temprana, algunos estudios han reportado que los niños y adolescentes con SM, tienen de 2 a 3 veces mayor riesgo de presentar en edad adulta SM, DM2 y engrosamiento de la íntima media de la carótida (19).

Varios marcadores clínicos y bioquímicos se han utilizado para predecir el riesgo cardiovascular (RCV). En los últimos años se ha prestado especial atención al índice Triglicéridos/HDL-Colesterol (TG/HDL-C) debido al papel preponderante en la fisiopatología del SM de dos alteraciones frecuentes: niveles elevados de triglicéridos (TG) y disminución del HDL-C. En los adultos este índice ha demostrado ser un marcador útil para predecir RI y mortalidad por ECV con un punto de corte $>3,5(20,21)$. También ha sido asociado con daño vascular desde edades tempranas de la vida (22). Por lo cual emerge como una alternativa útil y práctica para el diagnóstico de SM sobre el tradicional índice HOMA-IR (23). Otros investigadores han demostrado su utilidad como marcador de RCV en niños y adolescentes con puntos de corte más bajos que los utilizados en adultos $(24,25)$. En este contexto, el objetivo de nuestro estudio fue determinar la utilidad del índice TG/HDL-C para el diagnóstico de SM en niños y adolescentes obesos de 2 a 14 años de edad.

\section{MATERIAL Y MÉTODOS}

Estudio transversal, tipo evaluación de prueba de diagnóstico, que incluyó niños de ambos sexos de 2 a 14 años de edad con obesidad exógena, atendidos en la Unidad de Endocrinología Pediátrica del Hospital Cayetano Heredia (HCH), entre 2014 al 2018. No se calculó tamaño muestral, se incluyó a la población atendida en el periodo de estudio según los criterios de inclusión. Se definió obesidad exógena al exceso de grasa corporal secundaria al desequilibrio de la ingesta calórica y el gasto calórico, excluyendo niños con trastornos neuropsiquiátricos, enfermedades crónicas de compromiso sistémico, endocrinopatías, corticoterapia prolongada y síndromes dismórficos conocidos. El exceso de grasa corporal se determinó con el índice de masa corporal (IMC) mayor o igual a percentil 95 según los criterios del Center of Disease Control. 
Los pacientes seleccionados fueron distribuidos en 3 grupos etarios: 2 a 5 años, 6 a 9 años y 10 a 14 años. La evaluación antropométrica se realizó según los estándares de la OMS. Para medir el peso se utilizó una balanza marca SECA con precisión de 100 gramos. La presión arterial fue medida según recomendaciones de la AAP, el perfil lipídico y la glucosa se determinaron por método colorimétrico. Se definió SM, según la presencia de 3 o más criterios de la IDF, considerando obligatorio la circunferencia de cintura mayor a 90 percentil para edad y sexo. Se consideró al índice TG/HDL-C como factor de riesgo cardiovascular con dos puntos de corte: $\geq 2,32$ y $\geq 3,5$. Se comparó la frecuencia del índice como factor de riesgo según la presencia o ausencia de SM para cada punto de corte y grupo etario. Se aplicó Chi cuadrado, considerando significativo $\mathrm{p}<0,05$. Se determinó sensibilidad (S), especificidad (E), valor predictivo positivo (VPP), valor predictivo negativo (VPN), likelihood ratio positivo (LR+) y likelihood ratio negativo (LR-) por punto de corte y grupo etario. Fue utilizado el paquete estadístico STATA 15.
El estudio fue aprobado por el Comité de Ética del Hospital Cayetano Heredia.

\section{RESULTADOS}

Fueron incluidos 360 niños obesos, 55,2 \% fueron varones. Las características generales de la población de estudio se muestran en la tabla 1.

La frecuencia de SM fue $15,8 \%$ en niños de 2 a 5 años, $20,2 \%$ de 6 a 9 años y $19,6 \%$ de 10 a 14 años, sin diferencia significativa $(\mathrm{p}=0,66)$. La frecuencia de SM según sexo fue $24,2 \%$ en mujeres y $14,5 \%$ en varones $(\mathrm{p}=0,002)$.

La frecuencia de los componentes del SM en los niños según grupo etario, se pueden observar en la tabla 2; los componentes con mayor frecuencia fueron $\mathrm{TG} \geq 150 \mathrm{mg} / \mathrm{dl}$, HDL-C y $\mathrm{CC}>\mathrm{p} 90$.

Se encontró asociación significativa entre SM y el índice TG/HDL-C en ambos puntos de corte, en los tres grupos etarios (tabla 3 ).

Tabla 1. Características generales de la población de estudio.

\begin{tabular}{|c|c|c|c|c|c|c|}
\hline & \multicolumn{2}{|c|}{ 2-5 años (n=95) } & \multicolumn{2}{|c|}{ 6-9 años $(n=158)$} & \multicolumn{2}{|c|}{10 a 14 años $(n=107)$} \\
\hline & $F(n=43)$ & $M(n=52)$ & $\mathrm{F}(\mathrm{n}=74)$ & $M(n=84)$ & $F(n=44)$ & $M(n=63)$ \\
\hline z - IMC (DS) & $2,6 \pm 0,53$ & $3,39 \pm 0,77$ & $2,15 \pm 0,30$ & $2,25 \pm 0,32$ & $2,05 \pm 0,24$ & $2,12 \pm 0,26$ \\
\hline $\begin{array}{l}\text { Circunferencia cintura } \\
\qquad(\mathrm{cm})\end{array}$ & $69,85 \pm 9,04$ & $68,22 \pm 10,83$ & $78,06 \pm 8,29$ & $79,32 \pm 8$ & $87,02 \pm 5,45$ & $87,96 \pm 7,16$ \\
\hline Glucosa (mg/dl) & $83,22 \pm 8,28$ & $85,43 \pm 9,05$ & $88,42 \pm 8,55$ & $87,79 \pm 7,49$ & $90,57 \pm 5,67$ & $90,57 \pm 5,67$ \\
\hline $\begin{array}{l}\text { Presión arterial sistólica } \\
\qquad(\mathrm{mm} \mathrm{Hg})\end{array}$ & $92,58 \pm 13,98$ & $94,19 \pm 12,81$ & $90,66 \pm 8,7$ & $92,36 \pm 9,99$ & $99,5 \pm 9,09$ & $98,11 \pm 8,68$ \\
\hline $\begin{array}{l}\text { Presión arterial diastólica } \\
\qquad(\mathrm{mm} \mathrm{Hg})\end{array}$ & $59,79 \pm 10,15$ & $59,87 \pm 9,15$ & $57,46 \pm 7,76$ & $59,39 \pm 8,62$ & $63,52 \pm 5,39$ & $62,97 \pm 6,39$ \\
\hline TG/HDL-C & $3,01 \pm 2,11$ & $2,58 \pm 1,41$ & $3,74 \pm 2,47$ & $1,99 \pm 1,97$ & $3,99 \pm 2,38$ & $3,30 \pm 1,95$ \\
\hline
\end{tabular}

*Los datos son expresados en media y DS.

Tabla 2. Distribución de los componentes en pacientes con síndrome metabólico por grupo etario y sexo.

\begin{tabular}{ccccccc}
\hline & \multicolumn{2}{c}{$\mathbf{2 - 5}$ años } & $(\mathbf{n}=\mathbf{1 5})$ & \multicolumn{2}{c}{ 6-9 años $(\mathbf{n = 2 2})$} & \multicolumn{2}{c}{$\mathbf{1 0}$ a 14 años (n=21) } \\
\hline Componente & $\mathrm{F}$ & $\mathrm{M}$ & $\mathrm{F}$ & $\mathrm{M}$ & $\mathrm{F}$ & $\mathrm{M}$ \\
$\mathrm{CC} \geq \mathrm{p} 90$ & $(60 \%)$ & $(40 \%)$ & $(53,1 \%)$ & $(46,9 \%)$ & $(61,9 \%)$ & $(38,1 \%)$ \\
Triglicéridos $>150 \mathrm{mg} / \mathrm{dl}$ & 100 & 100 & 100 & 100 & 100 & 100 \\
HDL-C $<40 \mathrm{mg} / \mathrm{dl}$ & 77,78 & 66,67 & 82,35 & 80 & 100 & 87,50 \\
Glucosa $>100 \mathrm{mg} / \mathrm{dl}$ & 100 & 100 & 100 & 86,67 & 100 & 100 \\
$\mathrm{PAS} \geq 130 \mathrm{mmHg}$ & 0 & 33,33 & 35,29 & 40 & 7,69 & 12,50 \\
$\mathrm{PAD} \geq 85 \mathrm{mmHg}$ & 11,11 & 0 & 0 & 0 & 0 & 0 \\
\hline
\end{tabular}

Datos expresados en \%. CC: circunferencia de cintura PAS: presión arterial sistólica, PAD: presión arterial diastólica 
Tabla 3. TG/HDL- C y síndrome metabólico según punto de corte y grupo etario.

\begin{tabular}{|c|c|c|c|c|c|c|c|}
\hline \multirow{2}{*}{$\begin{array}{l}\text { Grupo } \\
\text { Etario } \\
\text { (años) }\end{array}$} & \multirow[t]{2}{*}{$\mathbf{n}$} & \multicolumn{3}{|c|}{ TG/HDL $\geq 2,32$} & \multicolumn{3}{|c|}{ TG/HDL $\geq \mathbf{3 , 5}$} \\
\hline & & $\begin{array}{c}\text { SM + } \\
(\%)\end{array}$ & $\begin{array}{c}\text { SM - } \\
(\%)\end{array}$ & $\mathbf{p}$ & $\begin{array}{c}\text { SM + } \\
(\%)\end{array}$ & $\begin{array}{c}\text { SM - } \\
(\%)\end{array}$ & p \\
\hline 2 a 5 & 95 & 86,7 & 43,8 & 0,002 & 80 & 20 & 0,001 \\
\hline 6 a 9 & 158 & 93,8 & 57,1 & 0,001 & 81,3 & 24,6 & 0,001 \\
\hline 10 a 14 & 107 & 100 & 54,7 & 0,001 & 100 & 27,9 & 0,001 \\
\hline
\end{tabular}

$\mathrm{SM}+$ : con SM, SM-: sin SM,

Tabla 4. Sensibilidad, especificidad, valor predictivo positivo, valor predictivo negativo y Likelihood Ratio según puntos de corte de TG/HDL y grupo etario.

\begin{tabular}{|c|c|c|c|c|c|c|}
\hline & \multicolumn{3}{|c|}{ TG/HDL $\geq 2,32$} & \multicolumn{3}{|c|}{ TG/HDL $\geq 3,5$} \\
\hline & $\begin{array}{l}2 \text { a } 5 \text { a } \\
(n=95)\end{array}$ & $\begin{array}{l}6 \text { a } 9 \text { a } \\
(n=158)\end{array}$ & $\begin{array}{l}10 \text { a } 14 \text { a } \\
(n=107)\end{array}$ & $\begin{array}{l}2 \text { a } 5 \text { a } \\
(n=95)\end{array}$ & $\begin{array}{l}6 \text { a } 9 \text { a } \\
(n=158)\end{array}$ & $\begin{array}{l}10 \text { a 14a } \\
(n=107)\end{array}$ \\
\hline SENSIBILIDAD & 86 & 94 & 100 & 80 & 81 & 100 \\
\hline ESPECIFICIDAD & 56 & 43 & 45 & 80 & 76 & 72 \\
\hline VPP (\%) & 27 & 29 & 30 & 43 & 46 & 46 \\
\hline VPN (\%) & 96 & 96 & 100 & 96 & 94 & 100 \\
\hline $\mathbf{L R}+$ & 1,95 & 1,64 & 1,81 & 4 & 3,38 & 3,57 \\
\hline LR- & 0,25 & 0,13 & 0 & 0,25 & 0,25 & 0 \\
\hline
\end{tabular}
negativo

La sensibilidad del índice fue alta para ambos puntos de corte, pero la especificidad fue mayor en el punto de corte $\geq 3,5$ (tabla 4). El VPN fue alto en ambos puntos de corte, en todas las edades. El LRtuvo tendencia a cero a mayor edad en ambos puntos de corte.

\section{DISCUSIÓN}

El incremento de la prevalencia de sobrepeso y obesidad en niños y adolescentes en las últimas dos décadas ha ocasionado un incremento de niños y adolescentes con SM, DM2 y riesgo cardiovascular. Varios marcadores bioquímicos han sido utilizados para el diagnóstico temprano de SM en niños y adolescentes; sin embargo, su difícil acceso por el costo y procesamiento ha impedido su uso generalizado. En éste contexto, el índice TG/HDL-C surge como un marcador útil en nuestro medio debido a su fácil accesibilidad y procesamiento.
En nuestro estudio, que incluyó niños obesos desde los 2 años de edad, encontramos una alta frecuencia en todos los grupos etarios de manera similar, pero la frecuencia de SM fue significativamente mayor en mujeres. Una revisión sistemática de la prevalencia de $\mathrm{SM}$, que incluyó predominantemente niños mayores de 6 años, tomando como referencia los criterios de la National Cholesterol Education Program's Adult Treatment Panel III (ATP), OMS y la IDF, reportó una prevalencia media de $29,2 \%$ en niños y adolescentes obesos, similar a nuestro estudio, pero con mayor frecuencia en varones y a mayor edad (26).

Muy pocos estudios han reportado la presencia de SM en niños menores de 6 años. Hirschler y col. (27), reportaron una prevalencia de 21,9\% (IC 95\% 12,24\%$31,0 \%$ ) en niños obesos con una edad promedio de $6,7 \pm 3$ años de edad, utilizando criterios de la ATP, generalmente utilizado en adultos. En nuestro estudio es importante resaltar la alta frecuencia de SM 
encontrada en los niños obesos del grupo etario de 2 a 5 años de edad, utilizando criterios considerados para niños mayores de 10 años de edad. Este hallazgo podría estar en relación al hallazgo de altos porcentajes de niños en todos los grupos etarios con niveles elevados de TG y niveles bajos de HDL-C, como ha sido reportado en otros estudios $(28,29)$ que podrían ser fácilmente detectados con la determinación del perfil lipídico.

La importancia y utilidad de los niveles elevados de TG y los niveles bajos de HDL-C en niños con $\mathrm{SM}$, evaluado como la relación o índice TG/HDL-C, ha sido reportado en niños y adolescentes de 6 a 16 años, encontrándose una sensibilidad de $80 \%$ y especificidad de $75 \%$ considerando un punto de corte $>1,25$; mientras el HOMA-IR considerando punto de corte $>2,76$ mostró una sensibilidad de $53,2 \%$ y especificidad de $69,5 \%$ (30).

Sin embargo, la información publicada sobre este índice en niños menores de 5 años es escasa. Nuestro estudio mostró alta sensibilidad en ambos puntos de corte y todas las edades, pero mayor especificidad en el punto de corte $\geq 3,5$ incluso en niños de 2 a 5 años, por lo que se podría suponer que las alteraciones de los triglicéridos y HDL-C expresado como índice TG/ HDL-C se presentan desde etapas tempranas de la vida.

La falta de estimación del tamaño de muestra y el sesgo de selección, constituyen limitaciones de este estudio, dado que el $\mathrm{HCH}$ es un establecimiento de referencia, donde se atienden pacientes con altos grados de obesidad, especialmente en los niños menores de 5 años de edad.

En conclusión, el índice TG/HDL-C $\geq 3,5$ representaría un marcador sensible y específico para el diagnóstico de síndrome metabólico desde los primeros años de vida y podría ser utilizado en la identificación precoz del SM en niños obesos.

\section{Declaración de financiamiento y conflicto de intereses:}

El estudio fue financiado por los autores y declaran no tener conflicto de intereses.

\section{Contribución de autoría:}

ECM: Diseño del estudio, recolección de datos, análisis e interpretación de resultados, elaboración del artículo, revisión crítica, aprobación de la versión final publicada; MMMP: Diseño del estudio, recolección de datos, análisis e interpretación de resultados, elaboración del artículo, revisión crítica, aprobación de la versión final publicada; YCC: Recolección de datos, análisis e interpretación de resultados, elaboración del artículo, aprobación de la versión final publicada; SMVP: Diseño del estudio, recolección de datos, análisis e interpretación de resultados, elaboración del artículo revisión crítica, aprobación de la versión final publicada; IGL: Recolección de datos, aprobación de la versión final publicada.

\section{Correspondencia:}

Emilio Cabello Morales

Dirección: Calle Ficaria 3849, Urb. Las Palmeras. Los Olivos.

Código Postal:15301

Correo electrónico: emilio.cabello@upch.pe

\section{REFERENCIAS BIBLIOGRÁFICAS}

1. Pajuelo J. La obesidad en el Perú. An Fac med. 2017; 78(2):179-185.

2. Falaschetti, E., Hingorani, A. D., Jones, A. et al. Adiposity and cardiovascular risk factors in a large contemporary population of pre-pubertal children. Eurheartj 010; 31 (24): 3063-3072.

3. Juonala M, Magnussen C, Berenson $G$, et al. Childhood adiposity, adult adiposity, and cardiovascular risk factors. N Engl J Med. 2011; 365 (20):1876-1885.

4. Spolidoro, J. V., Pitrez Filho, M. L., Vargas, L. T. et al. Waist circumference in children and adolescents correlate with metabolic syndrome and fat deposits in young adults. Clin Nutr. 2013; 32 (1): 93-97.

5. Rochlani, Y., Pothineni, N. V., Kovelamudi, S., \& Mehta, J. L. Metabolic syndrome: pathophysiology, management, and modulation by natural compounds. Ther Adv Cardiovasc Dis. 2017 Aug; 11(8):215-225.

6. Sinaiko AR, Steinberger J, Moran A et al. Relation of body mass index and insulin resistance to cardiovascular risk factors, inflammatory factors, and oxidative stress during adolescence. Circulation. 2005 Apr 19; 111(15):1985-91.

7. Steinberger J, Daniels SR, American Heart Association Atherosclerosis, Hypertension, and Obesity in the Young Committee, American Heart Association Diabetes Committee. Obesity, insulin resistance, diabetes, and cardiovascular risk in children: an American Heart Association scientific statement from the Atherosclerosis, Hypertension, and Obesity in the Young Committee (Council on 
Cardiovascular Disease in the Young) and the Diabetes Committee (Council on Nutrition, Physical Activity, and Metabolism). Circulation. 2003 Mar 18;107(10):1448-53

8. Tripathy, D., Mohanty, P., Dhindsa, S., et al. Elevation of free fatty acids induces inflammation and impairs vascular reactivity in healthy subjects. Diabetes. 2003; 52 (12): 2882-2887.

9. Hotamisligil, G. S., Murray, D. L., Choy, L. N., \& Spiegelman, B. M.. Tumor necrosis factor alpha inhibits signaling from theinsulinreceptor. Proceedings of the National Academy of Sciences. Proc Natl Acad Sci USA. 1994 May 24; 91(11):4854-8.

10. Lindsay RS, Funahashi T, Hanson RL et al. Adiponectin and development of type 2 diabetes in the Pima Indian population. Lancet. $2002 \mathrm{Jul} \mathrm{6;}$ 360(9326):57-8.

11. Vaněčková I, Maletínská L, Behuliak M et al. Obesityrelated hypertension: possible pathophysiological mechanisms. J Endocrinol. 2014 Dec; 223(3):R6378.

12. Magge SN, Goodman E, Armstrong SC, AAP COMMITTEE ON NUTRITION, SECTION ON ENDOCRINOLOGY, SECTION ON OBESITY. The Metabolic Syndrome in Children and Adolescents: Shifting the Focus to Cardiometabolic Risk Factor Clustering. Pediatrics. 2017;140(2): e20171603.

13. Expert Panel on Detection, Evaluation, and Treatment of High Blood Cholesterol in Adults. Executive Summary of the Third Report of the National Cholesterol Education Program (NCEP) Expert Panel on Detection, Evaluation, and Treatment of High Blood Cholesterol in Adults (Adult Treatment Panel III). JAMA. 2001;285(19):2486-2497.

14. Cook S, Weitzman M, Auinger P et al. Prevalence of a Metabolic Syndrome Phenotype in Adolescents Findings from the Third National Health and Nutrition Examination Survey, 1988-1994. Arch Pediatr Adolesc Med. 2003;157(8):821-827.

15. Cruz ML, Weigensberg MJ, Huang TT, Ball G, Shaibi GQ, Goran MI. The metabolic syndrome in overweight Hispanic youth and the role of insulin sensitivity. J Clin Endocrinol Metab. 2004; 89: 108-13.

16. Expert Panel on Integrated Guidelines for Cardiovascular Health and Risk Reduction in Children and Adolescents; National Heart, Lung, and Blood Institute. Expert Panel on Integrated Guidelines for Cardiovascular Health and Risk Reduction in children and adolescents: Summary Report. Pediatrics. 2011 Dec;128 Suppl 5:S213-56.

17. Zimmet P, Alberti G, Kaufman F, et al. International Diabetes Federation Task Force on Epidemiology and Prevention of Diabetes. The metabolic syndrome in children and adolescents. Lancet. 2007; 369: 20592061.
18. Cabello Morales, E.A.; Lozano Rojas, G.E.; Polar Córdova V., et al. Comparison of Criteria of the American Academy of Pediatrics (AAP) and the International Diabetes Federation (IDF) for the Diagnosis of Metabolic Syndrome and Its Relationship with HOMA-IR and Triglycerides/HDLc Ratio in Obese Children 4 to 14 Years Old. Horm Res Paeditr. 2014; 82 (suppl 2):25-45.

19. Magnussen, C. G., Koskinen, J., Chen, W., et al. Pediatric metabolic syndrome predicts adulthood metabolic syndrome, subclinical atherosclerosis, and type 2 Diabetes Mellitus but is no better than body mass index alone clinical perspective: The Bogalusa Heart Study and the Cardiovascular Risk in Young Finns Study. Circulation. 2010 Oct 19; 122(16):160411.

20. McLaughlin, T., Reaven, G., Abbasi, F., et al. Is there a simple way to identify insulin-resistant individuals at increased risk of cardiovascular disease? Am J Cardiol. 2005 Aug 1;96(3):399-404.

21. Vega GL, Barlow CE, Grundy SM, Leonard D, DeFina LF. Triglyceride-to-high-densitylipoprotein-cholesterol ratio is an index of heart disease mortality and of incidence of type 2 diabetes mellitus in men. J Investig Med. 2014 Feb; 62(2):3459.

22. de Giorgis, T., Marcovecchio, M. L., Di Giovanni, I., et al. Triglycerides-to-HDL ratio as a new marker of endothelial dysfunction in obese prepubertal children. Eur J Endocrinol. 2014 Dec 21;170(2):17380.

23. Cabello E, Lozano G., Cortez J., et al. Comparison of Three Diagnostic Definitions of Metabolic syndrome and their Relationship with the Homeostatic Model Assessment (HOMA) Index in Obese Children and Adolescents. Horm Res Paediatric.2011; 76 (suppl 4): 1-32

24. Di Bonito P, Moio N, Scilla C, et al. Usefulness of the High Triglyceride-to-HDL Cholesterol Ratio to Identify Cardiometabolic Risk Factors and Preclinical Signs of Organ Damage in Outpatient Children. Diabetes Care. 2012 Jan;35(1):158-62.

25. Lozano G, Cabello E, Polar V. Insulin Resistance Index (HOMA-IR) and Triglyceride/ HDLCholesterol Ratio as Cardiovascular Risk Markers in Obese Prepubertal and Pubertal Children. Horm Res Paeditr. 2014; 82 (suppl 2):1-45.

26. Friend A, Craig L, Turner S. The prevalence of metabolic syndrome in children: a systematic review of the literature. Metab Syndr Relat Disord. 2013 Apr; 11(2):71-80.

27. Hirschler V, Calcagno ML, Aranda C et al. Síndrome metabólico en la infancia y su asociación con insulinorresistencia. Arch Argent Pediatr. 2006; 104(6):486-491.

28. Sangun Ö, Dündar B, Köşker M et al. Prevalence of 
metabolic syndrome in obese children and adolescents using three different criteria and evaluation of risk factors. J Clin Res Pediatr Endocrinol. 2011; 3(2):706.

29. Gardner, A. W., Parker, D. E., Krishnan, S., \& Chalmers, L. J. et al. Metabolic syndrome and daily ambulation in children, adolescents, and young adults. Med Sci Sports Exerc. 2013 Jan; 45(1):163-9.
30. Liang J, Fu J, Jiang Y et al. Triglycerides and highdensity lipoprotein cholesterol ratio compared with homeostasis model assessment insulin resistance indexes in screening for metabolic syndrome in the chinese obese children: a cross section study. BMC Pediatr. 2015 Sep 28; 15: 138.

Recibido: 14/01/2019

Aceptado: 24/09/2019 